Pacific Journal of Mathematics

DECOMPOSITION OF HOLOMORPHS 


\section{DECOMPOSITION OF HOLOMORPHS}

\section{W. H. MILls}

Let $G$ be a group, and let $H$ be its holomorph. There are two situations in which $H$ is known to be decomposable into the direct product of two proper subgroups. If $G$ is the direct product of two of its proper characteristic subgroups, say $G_{1}$ and $G_{2}$, then $H$ is the direct product of the holomorphs of $G_{1}$ and $G_{2}$. If $G$ is a complete group, then $H$ is the direct product of $G$ and $G^{*}$, where $G^{*}$ is the centralizer of $G$ in $H$. In this paper we will show that if $G$ is not the direct product of two proper characteristic subgroups, and if $G$ is not complete, then $H$ is indecomposable. Thus we have a complete characterization of those groups whose holomorphs are indecomposable.

A decomposition of $H$ into the direct product of indecomposable factors is known for the case where $G$ is a finite abelian group [1]. Our present results enable us to generalize this and give a decomposition of $H$ into the direct product of indecomposable factors, whenever $G$ is the direct product of a finite number of characteristically indecomposable characteristic subgroups. In particular this gives a complete decomposition of $H$ whenever $G$ is a finite group.

Peremans [2] has shown that a necessary and sufficient condition for $G$ to be a direct factor of $H$ is that $G$ be either complete or the direct product of a group of order two and a complete group that has no subgroups of index two. This result is related to the present paper. In fact Peremans' result can be deduced from Lemma $1^{*}$.

1. Preliminaries. Let $G$ be a group, and let $A$ be the group of all automorphisms of $G$. Let $e$ and $I$ denote the identities of $G$ and $A$ respectively. The holomorph $H$ of $G$ can be regarded as the semi-direct product of $G$ and $A$, i.e., the set of all pairs $(g, \sigma), g \in G, \sigma \in A$, with multiplication defined by

$$
(g, \sigma)(h, \tau)=(g(\sigma h), \sigma \tau) .
$$

We identify $g$ in $G$ with $(g, I)$ in $H$. Then $H$ is a group that contains $G$ as an invariant subgroup, and every automorphism of $G$ can be extended to an inner automorphism of $H$.

For all $a$ in $G$ we let $\lambda_{a}$ denote the inner automorphism of $G$ corresponding to the element $a$. Thus $\lambda_{a} g=a g a^{-1}$.

All the results of this paper depend on the following lemma:

Lemma 1. Let $H=H_{1} \times H_{2}$. Then $G \cap H_{1}$ and $G \cap H_{2}$ are characteristic subgroups of $G$ and

Received November 1, 1960. 


$$
G=\left(G \cap H_{1}\right) \times\left(G \cap H_{2}\right)
$$

Proof. We note first that $G \cap H_{1}$ and $G \cap H_{2}$ are normal subgroups of $H$, and hence they are characteristic subgroups of $G$.

For $i=1$ or 2 , let $\varepsilon_{i}$ denote the projection of $H$ onto $H_{i}$ corresponding to the decomposition $H=H_{1} \times H_{2}$. Thus if $\alpha \in H_{1}$ and $\beta \in H_{2}$, then $\varepsilon_{1}(\alpha \beta)=\alpha$ and $\varepsilon_{2}(\alpha \beta)=\beta$. Put $J_{i}=\varepsilon_{i} G$. Clearly $J_{i} \subseteq H_{i}$ and $J_{i}$ is a normal subgroup of $H$. Let $F_{i}$ and $S_{i}$ denote the set of all first and second components respectively of elements of $J_{i}$. Thus $F_{i} \subseteq G$ and $S_{i} \leqq A$.

Let $(a, \sigma)$ be an element of $J_{1}$. Then for some $g$ in $G$ we have $\varepsilon_{1} g=(a, \sigma)$. Put $\varepsilon_{2} g=(b, \tau)$. Then $g=(a, \sigma)(b, \tau)$. Therefore $\tau=\sigma^{-1}$ and $\left(\sigma b^{-1}, \sigma\right)=(b, \tau)^{-1} \in J_{2}$. Hence $\sigma \in S_{2}$. It follows that $S_{1} \leqq S_{2}$. By symmetry $S_{2} \subseteq S_{1}$, and hence $S_{1}=S_{2}$.

Let $\sigma$ be an element of $S_{1}$ and let $\xi$ be an element of $A$. Put $\varepsilon_{i}(e, \xi)=\left(g_{i}, \xi_{i}\right), i=1,2$. For some $a$ and $c$ in $G$ we have $(a, \sigma) \in J_{1}$ and $(c, \sigma) \in J_{2}$. Now

$$
(a, \sigma)\left(g_{2}, \xi_{2}\right)=\left(g_{2}, \xi_{2}\right)(a, \sigma)
$$

and

$$
(c, \sigma)\left(g_{1}, \xi_{1}\right)=\left(g_{1}, \xi_{1}\right)(c, \sigma) .
$$

Comparing second components we see that $\sigma$ commutes with both $\xi_{1}$ and $\xi_{2}$. Since $\xi=\xi_{1} \xi_{2}$, we have $\sigma \xi=\xi \sigma$. It follows that $S_{1}$ is contained in the center of $A$.

Let $(a, \sigma)$ be an element of $J_{1}$ and let $(d, \mu)$ be an element of $J_{2}$. Since $\sigma$ is contained in the center of $A$ and since $(a, \sigma)^{-1}=\left(\sigma^{-1} a^{-1}, \sigma^{-1}\right)$, it follows that

$$
d(a, \sigma) d^{-1}\left(e, \lambda_{\sigma d}\right)(a, \sigma)^{-1}\left(e, \lambda_{\sigma d}\right)^{-1}=d(\sigma d)^{-1} .
$$

Therefore $d(\sigma d)^{-1} \in H_{1}$. Moreover

$$
d(\sigma d)^{-1}=(d, \mu)(e, \sigma)(d, \mu)^{-1}(e, \sigma)^{-1} \in H_{2} .
$$

Hence $d(\sigma d)^{-1} \in H_{1} \cap H_{2}$. This gives us $d(\sigma d)^{-1}=e$ and $\sigma d=d$. Thus $\sigma$ leaves every element of $F_{2}$ fixed. By symmetry, since $\sigma \in S_{1}=S_{2}$, it follows that $\sigma$ leaves every element of $F_{1}$ fixed. Now let $g$ be an arbitrary element of $G$. Then $g=(f, \nu)(h, \zeta)$ with $(f, \nu) \in J_{1}$ and $(h, \zeta)$ $\in J_{2}$. Since $g=f(\nu h), \sigma f=f$, and $\sigma \nu h=\nu \sigma h=\nu h$, it follows that $\sigma g=g$. Hence $\sigma=I$. Therefore $S_{1}$ and $S_{2}$ consist of the identity alone. It follows that $J_{1} \subseteq G \cap H_{1}, J_{2} \subseteq G \cap H_{2}$, and

$$
G \subseteq J_{1} \times J_{2} \subseteq\left(G \cap H_{1}\right) \times\left(G \cap H_{2}\right) \subseteq G .
$$

Therefore $G=\left(G \cap H_{1}\right) \times\left(G \cap H_{2}\right)$ and the proof is complete. 
2. Some known results. Suppose $G=G_{1} \times G_{2} \times \cdots \times G_{n}$, where the $G_{i}$ are characteristic subgroups of $G$. Let $A_{i}$ denote the group of all automorphisms of $G_{i}$. We identify $\sigma_{i}$ in $A_{i}$ with the element $\sigma_{i}^{\prime}$ in $A$ such that

$$
\sigma_{i}^{\prime} g= \begin{cases}g & \text { if } g \in G_{j}, j \neq i \\ \sigma_{i} g & \text { if } g \in G_{i} .\end{cases}
$$

Then $A=A_{1} \times A_{2} \times \cdots \times A_{n}$. Moreover $H_{i}$, the holomorph of $G_{i}$, becomes a subgroup of $H$, and $H=H_{1} \times H_{2} \times \cdots \times H_{n}$.

The centralizer of a group in its holomorph is called its conjoint. The conjoint $G^{*}$ of $G$ consists of the elements $\left(g^{-1}, \lambda_{q}\right), g \in G$. The mapping $\eta$ defined by

$$
\eta(g, \sigma)=\left(g^{-1}, \lambda_{\rho} \sigma\right)
$$

is an automorphism of $H$ that maps $G$ onto $G^{*}$ and maps $G^{*}$ onto $G$. Therefore $G$ and $G^{*}$ are isomorphic, and $G$ is the centralizer of $G^{*}$ in $H$. Furthermore Lemma 1 is equivalent to the following:

Lemma $1^{*}$. Let $H=H_{1} \times H_{2}$. Then $G^{*} \cap H_{1}$ and $G^{*} \cap H_{2}$ are characteristic subgroups of $G^{*}$ and

$$
G^{*}=\left(G^{*} \cap H_{1}\right) \times\left(G^{*} \cap H_{2}\right) .
$$

If $G$ is complete, i.e., if $G$ is a centerless group with only inner automorphisms, then $H=G \times G^{*}$.

3. Decomposable and indecomposable holomorphs. If $G$ is the direct product of two proper characteristic subgroups, then $G$ is said to be characteristically decomposable. If not, then $G$ is said to be characteristically indecomposable.

THeOREM 1. Let $G$ be a group, and let $H$ be its holomorph. If $G$ is either characteristically decomposable or complete, then $H$ is decomposable. If $G$ is characteristically indecomposable and not complete, then $H$ is indecomposable.

Proof. We have seen in $\S 2$ that $H$ is decomposable if $G$ is either characteristically decomposable or complete. Suppose that $G$ is characteristically indecomposable and that $H=H_{1} \times H_{2}$. It follows from Lemma 1 that either $G \cap H_{1}=G$ or $G \cap H_{2}=G$. Thus either $G \cong H_{1}$ or $G \leqq H_{2}$. Similarly it follows from Lemma $1^{*}$ that either $G^{*} \cong H_{1}$ or $G^{*} \subseteq H_{2}$. Without loss of generality suppose that $G \leqq H_{1}$. Then $H_{2}$ is contained in the centralizer of $G$, that is $H_{2} \subseteq G^{*}$. If $G^{*} \subseteq H_{1}$ we have $H_{2} \subseteq H_{1}$ and $H=H_{1}$. Thus we need only consider the case $G^{*} \cong H_{2}$. 
Here $G^{*}=H_{2}$ and $H_{1}$ is contained in the centralizer of $G^{*}$. Thus $H_{1} \leqq G$, and hence $H_{1}=G$. Now $G \cap G^{*}$ is the center of $G$, and $G \cap G^{*}=$ $H_{1} \cap H_{2}$. Hence $G$ is centerless. Since $H=H_{1} \times H_{2}=G \times G^{*}$, it follows that $G$ has only inner automorphisms. Therefore $G$ is complete. This completes the proof of the theorem.

4. Decomposition of the holomorph into indecomposable subgroups. To complete our discussion we need the following result:

LEMMA 2. If a group is complete and characteristically indecomposable, then it is indecomposable.

Proof. Let $G$ be a complete group and suppose $G=G_{1} \times G_{2}$. Since every automorphism of $G$ is inner, it follows that every automorphism of $G$ maps $G_{1}$ and $G_{2}$ onto themselves. Hence $G_{1}$ and $G_{2}$ are characteristic subgroups of $G$. This establishes the lemma.

THEOREM 2. Suppose $G$ is the direct product of a finite number of characteristically indecomposable characteristic subgroups: $G=$ $G_{1} \times G_{2} \times \cdots \times G_{n}$. Suppose that $G_{i}$ is complete for $1 \leqq i \leqq r$, and that $G_{j}$ is not complete for $r+1 \leqq j \leqq n$. Then a decomposition of $H$ into indecomposable subgroups is given by

$$
H=\prod_{i=1}^{r} G_{i} \times \prod_{i=1}^{r} G_{i}^{*} \times \prod_{i=r+1}^{n} H_{i},
$$

where $G_{i}^{*}$ and $H_{i}$ are the conjoint and holomorph respectively of $G_{i}$, and where $I$ denotes a direct product.

Proof. It follows from $\S 2$ that (1) is a decomposition of $H$. By Lemma 2 the groups $G_{i}$ and $G_{i}^{*}$ are indecomposable for $1 \leqq i \leqq r$, and by Theorem 1 the groups $H_{i}$ are indecomposable for $r+1 \leqq i \leqq n$.

Since a characteristic subgroup of a characteristic subgroup of $G$ is itself a characteristic subgroup of $G$ it follows that $G$ satisfies the condition of Theorem 2 whenever the characteristic subgroups of $G$ satisfy the descending chain condition. In particular Theorem 2 gives us a decomposition of $H$ into indecomposable subgroups whenever $G$ is a finite group.

If $G$ is the direct product of an infinite number of characteristic subgroups, then $H$ is not the direct product of their holomorphs. Thus Theorem 2 does not hold in this case.

\section{BIBLIOGRAPHY}

1. W. H. Mills, On the non-isomorphism of certain holomorphs, Trans. Amer. Math. Soc., 74 (1953), 428-443.

2. W. Permans, Completeness of holomorphs, Nederl. Akad. Wetensch. Proc. Ser. A 60 (1957), 608-619. 


\section{PACIFIC JOURNAL OF MATHEMATICS}

\section{EDITORS}

\author{
RaLPh S. Phillips \\ Stanford University \\ Stanford, California \\ F. H. BRowNELL \\ University of Washington \\ Seattle 5 , Washington
}

A. L. Whiteman

University of Southern California

Los Angeles 7, California

L. J. Paige

University of California

Los Angeles 24, California

\author{
E. F. BECKENBACH \\ T. M. CHERRY
}

\author{
ASSOCIATE EDITORS

$\begin{array}{lll}\text { D. DERRY } & \text { H. L. ROYDEN } & \text { E. G. STRAUS } \\ \text { M. OHTSUKA } & \text { E. SPANIER } & \text { F. WOLF }\end{array}$

\section{SUPPORTING INSTITUTIONS}

\author{
UNIVERSITY OF BRITISH COLUMBIA \\ CALIFORNIA INSTITUTE OF TECHNOLOGY \\ UNIVERSITY OF CALIFORNIA \\ MONTANA STATE UNIVERSITY \\ UNIVERSITY OF NEVADA \\ NEW MEXICO STATE UNIVERSITY \\ OREGON STATE COLLEGE \\ UNIVERSITY OF OREGON \\ OSAKA UNIVERSITY \\ UNIVERSITY OF SOUTHERN CALIFORNIA
}

\author{
STANFORD UNIVERSITY \\ UNIVERSITY OF TOKYO \\ UNIVERSITY OF UTAH \\ WASHINGTON STATE COLLEGE \\ UNIVERSITY OF WASHINGTON \\ AMERICAN MATHEMATICAL SOCIETY \\ CALIFORNIA RESEARCH CORPORATION \\ HUGHES AIRCRAFT COMPANY \\ SPACE TECHNOLOGY LABORATORIES \\ NAVAL ORDNANCE TEST STATION
}

Mathematical papers intended for publication in the Pacific Journal of Mathematics should be typewritten (double spaced), and the author should keep a complete copy. Manuscripts may be sent to any one of the four editors. All other communications to the editors should be addressed to the managing editor, L. J. Paige at the University of California, Los Angeles 24, California.

50 reprints per author of each article are furnished free of charge; additional copies may be obtained at cost in multiples of 50 .

The Pacific Journal of Mathematics is published quarterly, in March, June, September, and December. The price per volume (4 numbers) is $\$ 12.00$; single issues, $\$ 3.50$. Back numbers are available. Special price to individual faculty members of supporting institutions and to individual members of the American Mathematical Society: $\$ 4.00$ per volume; single issues, $\$ 1.25$.

Subscriptions, orders for back numbers, and changes of address should be sent to Pacific Journal of Mathematics, 103 Highland Boulevard, Berkeley 8, California.

Printed at Kokusai Bunken Insatsusha (International Academic Printing Co., Ltd.), No. 6, 2-chome, Fujimi-cho, Chiyoda-ku, Tokyo, Japan.

\section{PUBLISHED BY PACIFIC JOURNAL OF MATHEMATICS, A NON-PROFIT CORPORATION}

The Supporting Institutions listed above contribute to the cost of publication of this Journal, but they are not owners or publishers and have no responsibility for its content or policies.

Reprinted 1966 in the United States of America 


\section{Pacific Journal of Mathematics}

\section{Vol. 11, No. 4}

A. V. Balakrishnan, Prediction theory for Markoff processes . . . . . . . . . . 1171

Dallas O. Banks, Upper bounds for the eigenvalues of some vibrating systems . . . . 1183

A. Białynicki-Birula, On the field of rational functions of algebraic groups ...... 1205

Thomas Andrew Brown, Simple paths on convex polyhedra .............. 1211

L. Carlitz, Some congruences for the Bell polynomials . . . . . . . . . . . . 1215

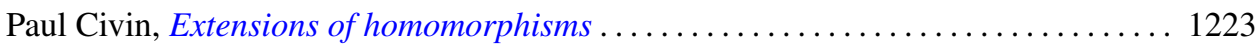

Paul Joseph Cohen and Milton Lees, Asymptotic decay of solutions of differential

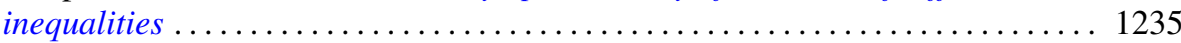

István Fáry, Self-intersection of a sphere on a complex quadric . . . . . . . . . . 1251

Walter Feit and John Griggs Thompson, Groups which have a faithful representation

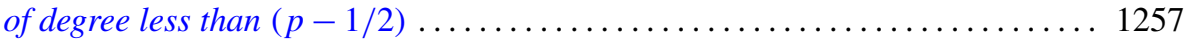

William James Firey, Mean cross-section measures of harmonic means of convex

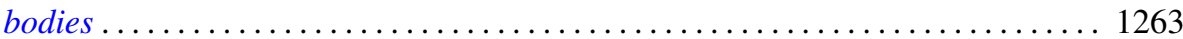

Avner Friedman, The wave equation for differential forms . . . . . . . . . . 1267

Bernard Russel Gelbaum and Jesus Gil De Lamadrid, Bases of tensor products of

Banach spaces ................................... 1281

Ronald Kay Getoor, Infinitely divisible probabilities on the hyperbolic plane . . . . 1287

Basil Gordon, Sequences in groups with distinct partial products . . . . . . . . . . . . 1309

Magnus R. Hestenes, Relative self-adjoint operators in Hilbert space . . . . . . . . . 1315

Fu Cheng Hsiang, On a theorem of Fejér ......................... 1359

John McCormick Irwin and Elbert A. Walker, On N-high subgroups of Abelian

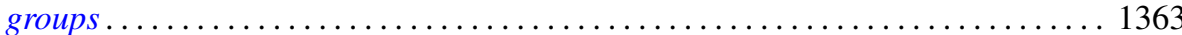

John McCormick Irwin, High subgroups of Abelian torsion groups . . . . . . . . . 1375

R. E. Johnson, Quotient rings of rings with zero singular ideal . . . . . . . . . . . 1385

David G. Kendall and John Leonard Mott, The asymptotic distribution of the time-to-escape for comets strongly bound to the solar system ...

Kurt Kreith, The spectrum of singular self-adjoint elliptic operators ....

Lionello Lombardi, The semicontinuity of the most general integral of the calculus of variations in non-parametric form ................................

Albert W. Marshall and Ingram Olkin, Game theoretic proof that Chebyshev inequalities are sharp

Wallace Smith Martindale, III, Primitive algebras with involution . . William H. Mills, Decomposition of holomorphs ..............

James Donald Monk, On the representation theory for cylindric algebras . . . . . . 1447

Shu-Teh Chen Moy, A note on generalizations of Shannon-McMillan theorem . . . . 1459

Donald Earl Myers, An imbedding space for Schwartz distributions . .

John R. Myhill, Category methods in recursion theory .........

Paul Adrian Nickel, On extremal properties for annular radial and circular slit mappings of bordered Riemann surfaces

Edward Scott O'Keefe, Primal clusters of two-element algebras . .

Nelson Onuchic, Applications of the topological method of Wazewski to certain

problems of asymptotic behavior in ordinary differential equations ...

Peter Perkins, A theorem on regular matrices................

Clinton M. Petty, Centroid surfaces .... 